\title{
LA REGIÓN FRONTERIZA MÉXICO-ESTADOS UNIDOS, UN LUGAR DE UNIÓN Y DESENCUENTROS
}

\author{
Manuel Mollá Ruiz-Gómez \\ Departamento de Geografía \\ Universidad Autónoma de Madrid
}

\begin{abstract}
RESUMEN
El límite fronterizo que separa a México de Estados Unidos es mucho más que una línea de división y de conflictos. Es una región integrada, a pesar de las dificultades, bien delimitada geográficamente y con una serie de intereses e instituciones comunes que hacen que, desde la Geografía, tenga un notable interés. La línea divisoria que une y separa a, según muchos autores, el primer del tercer mundo, pero que comparten dos países que firmaron un tratado de libre comercio y que para México supone un acuerdo con el país que es su mayor socio comercial. Ver cómo funciona esa frontera y su correspondiente región es el principal objetivo de este artículo.
\end{abstract}

Palabras clave: frontera, región, acuerdos, conflictos, México, Estados Unidos.

\begin{abstract}
The borderline that separates Mexico from the United States is much more than a line of division and conflict. It is an integrated region, despite the difficulties, well defined geographically, and with a number of common interests and institutions that make it to have a great interest from a geographic point of view. Two countries that signed a free trade agreement share the line that joins and separates, according to many authors, the First and the Third World, and it is for Mexico an agreement with the country that is its most important trading partner. To see how that boundary and the corresponding region work is the main objective of this paper.
\end{abstract}

Key words: Borderline, Region, Agreements, Conflicts, Mexico, United States.

\section{Introducción}

Cuando se habla de la frontera entre México y Estados Unidos lo primero que suele venir a la cabeza es el doble problema de la emigración ilegal y el vergonzante «muro de la tortilla», y el del narcotráfico desde México a Estados Unidos y su contrapartida, el tráfico de armas, 
esta vez, en sentido norte-sur. También es una frontera que se suele definir por ser la de más tráfico de personas del mundo o por ser la única que separa al primer del tercer mundo. Si bien todos estos asuntos son importantes, no lo es menos el hecho de que también se habla de un concepto fundamental en la Geografía, el de región. En efecto, hay una visión regional que implica a estados de ambos países, con sus ventajas y sus inconvenientes, que está bien definida por acuerdos internacionales entre México y Estados Unidos.

Es cierto que los problemas arriba indicados son de una notable repercusión para los dos países y para el mundo en general. La guerra que el actual gobierno de México mantiene contra el narcotráfico con sus miles de muertos; las quejas de este país por la «fluidez» con que las armas entran para los carteles desde el norte; la explotación de los inmigrantes ilegales por los grupos de traficantes de personas; las víctimas que se producen en ese difícil tránsito fronterizo... son cuestiones que se tratan casi a diario en la prensa internacional; sin olvidar las negativas repercusiones que todo ello tiene en la importante industria estadounidense, la maquila, instalada en los estados mexicanos fronterizos, que ocupan a miles de personas y que ven cómo muchas de estas empresas buscan otros países más seguros o donde la mano de obra resulte más barata.

Por esta razón, por ser un asunto muy actual y bien conocido, ha parecido más interesante centrarse en la cuestión regional, es decir, en cómo, a pesar de todo, se puede hablar de un región fronteriza entre ambos países con una serie de mecanismos de funcionamiento y regulación comunes que repercuten en diez estados de ambas federaciones (cuatro de los Estados Unidos y seis de México).

\section{La región fronteriza}

Hablar de región fronteriza entre México y Estados Unidos es hablar de dos niveles de integración, uno definido por acuerdo entre ambos países y, como consecuencia de ello, un segundo nivel que comprende los diez estados antes mencionados.



Figura 1. La región fronteriza según el Acuerdo de 1983.

Fuente: Cooperación fronteriza e investigación científica. Embajada de los Estados Unidos en México. 
El 14 de agosto de 1983, en la ciudad de La Paz, Baja California Sur, se firmó un acuerdo (Acuerdo de La Paz / La Paz Agreement) entre los dos países y cuyo objetivo fundamental era la cooperación para la protección y mejora del medio ambiente en la zona fronteriza. El artículo 4 de dicho acuerdo establece que la zona fronteriza tendrá un ancho de 100 kilómetros a ambos lados de la frontera, tanto terrestre (3.141 kilómetros) como marítima. Esta disposición afecta a un total de diez estados (California, Arizona, Nuevo México y Texas, al norte, y Baja California, Sonora, Chihuahua, Coahuila, Nuevo León y Tamaulipas, al sur), lo que incluye 15 ciudades hermanas (Tijuana-San Diego; Mexicali-Calexico; San Luis-Yuma; Nogales-Nogales; Naco-Naco; Agua Prieta-Douglas; Puerto Palomas-Columbus; Ciudad Juárez-El Paso; Ojinaga-Presidio; Ciudad Acuña-Del Río; Piedras Negras-Eagle Pass; Nuevo Laredo-Laredo; Río Bravo-Weslaco; Reynosa-MacAllen y Matamoros-Brownsville). Además, se incluyen 25 condados estadounidenses, 35 municipios mexicanos y treinta y tres tribus indígenas ( 7 del lado mexicano y 26 del estadounidense), con un total de más de 13 millones de personas (según fuentes de 2005).

Los aspectos fundamentales del Acuerdo quedan bien recogidos en el artículo 1 del mismo, que dice:

ARTICLE 1. The United States of America and the United Mexican States, hereinafter referred to as the Parties, agree to cooperate in the field of environmental protection in the border area on the basis of equality, reciprocity and mutual benefit. The objectives of the present Agreement are to establish the basis for cooperation between the Parties for the protection, improvement and conservation of the environment and the problems which affect it, as well as to agree on necessary measures to prevent and control pollution in the border area, and to provide the framework for development of a system of notification for emergency situations. Such objectives shall be pursued without prejudice to the cooperation which the Parties may agree to undertake outside the border area. (Acuerdo de La Paz: 1983).

Al margen de la importancia de un acuerdo de estas características para la protección ambiental, la nueva región delimitada va a tener otras consecuencias que afectarán a las poblaciones de ambos lados de la frontera, tales como la libre circulación de sus habitantes o la elección del lugar de residencia; la libertad para trabajar a ambos lados de la misma o, en el caso mexicano, deducciones fiscales, como por ejemplo, la compra de coches de segunda mano en el lado estadounidense. Pese a todo, la tarea de investigar en los aspectos sociales de dicha integración no es fácil. Como señala Cristóbal Mendoza:

«La región fronteriza México-Estados Unidos es, en muchos aspectos, única. La demografía no es una excepción. En efecto, la frontera internacional imprime «personalidad» a la estructura y cambio de la población en el área. Sin embargo, concretar esa «personalidad» en variables comparables a ambos lados de la frontera, o conceptualizarla en un marco teórico consistente, no es fácil. Esta tarea viene dificultada por las fuentes estadísticas (censos y encuestas), en muchos casos elaboradas por organismos oficiales de ambos países, que se centran exclusivamente en México y Estados Unidos. Solo en el caso de algunas pocas encuestas que abordan problemáticas muy concretas y referidas a realidades geográficas muy limitadas se ha aplicado la misma metodología a la hora de levantar una encuesta binacional (por ejemplo, Vásquez Galán y Cueva Luna, 2001, para un estudio de caso de la región Matamoros-Brownsville)». (Mendoza, 
2001: 31-32). En general, se cuenta exclusivamente con los informes hechos por la SEMARNAT (Secretaría de Medio Ambiente y Recursos Naturales) de México, y la EPA (Environmental Protection Agency) de Estados Unidos, publicados con posterioridad al trabajo de Cristóbal Mendoza.

Lo mismo se podría decir de los censos que elaboran ambos países, puesto que los objetivos del INEGI mexicano (Instituto Nacional de Estadística, Geografía e Informática) y del US Census Bureau responden a intereses diferentes. Esto no impide, en cualquier caso, realizar estudios integrados y comparados entre ambos lados de la frontera, si bien es verdad que, hoy día, la guerra contra el narcotráfico no hace de esa región el lugar más seguro para los investigadores. El desequilibrio económico entre ambos países provoca que en esta región fronteriza los condicionamientos sean mucho más fuertes para el vecino del sur que del norte, como se puede comprobar cuando se analiza la evolución económica y demográfica de la región. Para México, la presencia de las maquiladoras, el deseo de cruzar la frontera de miles de sus ciudadanos y de países de América Central, ilegales también en México, entre otras, hacen que los cambios en la región sean mucho más fuertes que para los Estados Unidos. Bastaría señalar el caso de los miles de ciudadanos que se ven obligados a vivir en condiciones muy precarias en el entorno de ciudades como Tijuana o Ciudad Juárez, por señalar un par de ellas, como bien ha estudiado, entre otros autores, Isabel Rodríguez Chumillas. Para los Estados Unidos, además de la instalación de maquiladoras al sur de la frontera, tiene especial relevancia la llegada de braceros a las ricas tierras agrícolas de California y de los demás estados fronterizos, entre otras ventajas.

Por lo que se refiere a la actividad entre ambos lados de la frontera, según información dada por la Conferencia Legislativa Fronteriza, en 2008 la cruzaron casi 5 millones de camiones de carga y más de 105 millones de personas. Además, en esta región se encuentran las dos mayores metrópolis internacionales del mundo, Tijuana-San Diego, con unos cinco millones de habitantes, y la de Ciudad Juárez-El Paso, con unos 2,5 millones.

La existencia de esta región fronteriza, además de los programas de cooperación específicos de los que se hablará más adelante, permite hablar de un segundo nivel regional de mayor entidad en cuanto que implica a la totalidad de los estados afectados a ambos lados de la frontera. Es decir, un nivel formado ya por los diez estados y que pone en relación a las autoridades de los mismos con instituciones diferentes a las dos que se ocupan de los problemas ambientales de esa región de 100 kilómetros de ancho, que son la SEMARNAT y la EPA.

Este segundo nivel regional estaría representado por la Conferencia Legislativa Fronteriza (Border Legislative Conference) y que integra a los diez estados. Dicha Conferencia es un programa binacional que administran, por los Estados Unidos, el Consejo de Gobiernos Estatales del Oeste (CSG-WEST, en inglés) y por México, el Consejo Legislativo del Sur (CLS; SLC, en inglés). Su objetivo fundamental es la cooperación y el diálogo entre legisladores estatales de México y Estados Unidos, con el propósito de hacer que estos estados fronterizos fortalezcan sus relaciones y participen en la agenda binacional para el desarrollo de políticas públicas entre ambos países.

Tal y como lo señala la propia CLF en su página oficial,

«La CLF promueve el desarrollo de soluciones compartidas a lo largo de la región fronteriza a través de consideraciones conjuntas a problemas comunes y el intercambio de información. La CLF también busca establecer colaboraciones estratégicas con los diferentes niveles de gobierno y organizaciones no-gubernamentales con el fin que las comunidades fronterizas y gobiernos 


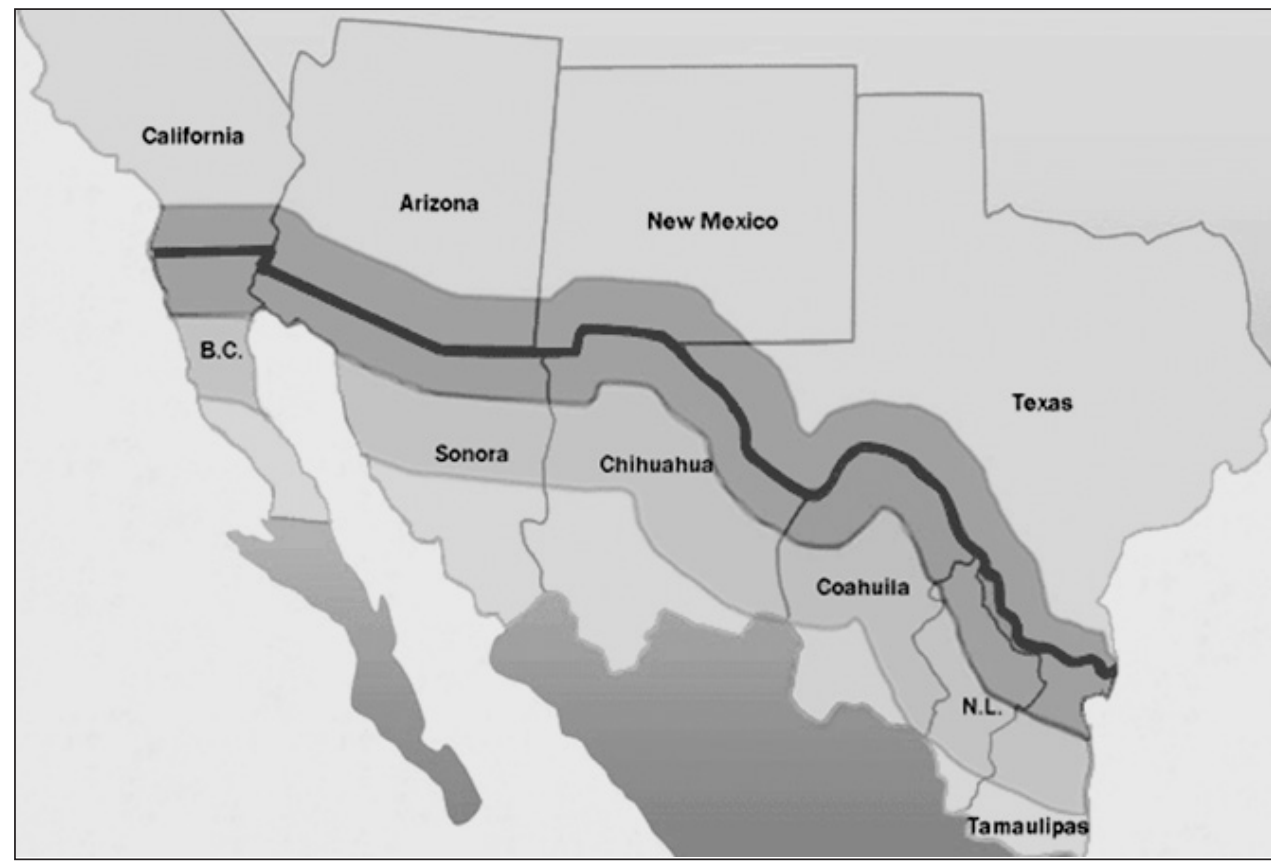

FIGURA 2. La región fronteriza entendida en sentido amplio.

Fuente: Conferencia Legislativa Fronteriza.

estatales de ambos lados de la frontera fortalezcan y mejoren.» (Conferencia Legislativa Fronteriza).

El apoyo económico lo proporciona la Agencia de Estados Unidos para el Desarrollo Internacional (USAID, en inglés).

El papel de los asociados, es decir, el CSG-WEST, el CLS y la USAID, como responsables de esta gran conferencia regional, viene determinado en misma página oficial.

«El Consejo de Gobiernos Estatales-OESTE (CSG-WEST) provee una plataforma no-partidista para cooperación regional entre las legislaturas del Oeste de los EEUU creando oportunidades para legisladores y sus empleados a compartir ideas y experiencias y vínculos institucionales con otros líderes políticos de la región. Las conferencias de CSG-WEST son abiertas a todos los legisladores, empleados legislativos, sector privado, y el público al pagar los costos de la conferencia que ayudan a compensar los costos directos para el encuentro. Esto incluye encuentros del comité ejecutivo de CSG-WEST; sin embargo, votos políticos, organizacionales y en asuntos son limitados a los legisladores de la región del Oeste que fueron designados por sus respectivas autoridades de cada cámara.»

«El objetivo de la USAID en respecto a México es soportar la cooperación bilateral para resolver los desafíos que enfrentan los dos países. Los programas 
de USAID apoyan a las iniciativas Mexicanas en su reforma del sistema judicial, la competitividad y el desarrollo sostenible a fin de que México pueda ofrecer a sus ciudadanos oportunidades económicas y comunidades seguras. Actividades conjuntas de desarrollo aumentan el entendimiento entre nuestros dos pueblos y muestra las oportunidades más allá de las fronteras de México para la cooperación Sur-Sur.»

«La misión del Consejo Legislativo del Sur es de fomentar cooperación intergubernamental entre los 15 estados miembros. En gran parte esto se consigue por conferencias, publicaciones, y posiciones políticas de las seis comisiones permanentes del Consejo. Miembros del Consejo son nombrados por el liderazgo legislativo de cada estado y cada comisión elige a sus oficiales. Por las deliberaciones de los miembros de la comisión, surge una lista de los asuntos que confrontan todas las legislaturas de los estados del sur. Cargado inicialmente con servir las tres ramas del gobierno estatal, las funciones de la oficina se han concretado en proveer servicios primordialmente a los más de 2,400 miembros legislativos y sus empleados de la región de 15 estados. Miembros del Consejo Legislativo del Sur son nombrados por el liderazgo de las 30 cámaras legislativas del sur.» (Conferencia Legislativa Fronteriza).

Como se puede apreciar, hay una discrepancia entre el número de estados mexicanos que participan en la CLF (6) y en el CLS (15). Esto se debe a que, además de los legisladores de los estados fronterizos, miembros de pleno derecho, se pueden incorporar otros estados de ambos países, admitidos como «miembros afiliados» por consenso de los diez estados fronterizos, aunque sin derecho a voto (la mayoría se obtiene, entre los diez estados, con cuatro estados mexicanos y tres estadounidenses).

Por último, señalar en este apartado que las competencias de la CLF no son exactamente las mismas que las que marca el Acuerdo de La Paz, con competencias exclusivas en asuntos de medio ambiente. En la Conferencia, además de los temas medioambientales y del agua, se incluyen todos los aspectos que pueden afectar a la región, tales como desarrollo económico, salud, cruces fronterizos, tráfico ilegal y seguridad.

\section{La cooperación regional}

De nuevo, como se acaba de señalar, la cooperación fronteriza tiene dos niveles de actuación. El primero de ellos es el que se determinó en el Acuerdo de La Paz entre los gobiernos de México y de Estados Unidos, relativo al medio ambiente y organizado en programas.

En la actualidad, el programa marco de las relaciones fronterizas desarrolladas por la SEMARNAT y por la EPA, es decir, las autoridades federales de ambos países en el campo ambiental, se conoce con el nombre «Frontera 2012: programa ambiental México-Estados Unidos (U.S.-Mexico Enviromental Program - Border 2012-). Dicho documento marco (a diez años) se firmó el 4 de abril de 2003 y recoge las bases para la cooperación en asuntos de medio ambiente entre ambos países y aplicados a la región fronteriza.

El programa se basa en la necesidad de proteger la salud de los ya más de 13 millones de habitantes (sólo un 10\% vive en áreas rurales) de la franja fronteriza de 100 kilómetros, mediante una cooperación binacional centrada en la limpieza del aire, la provisión de agua potable, reducción del riesgo a la exposición de sustancias peligrosas y la creación de una eficaz red de emergencias a lo largo de la frontera. 
En el mismo se reconoce la variedad paisajística, con amplias áreas desérticas, conjuntos montañosos, ríos, humedales, grandes estuarios y acuíferos compartidos. Pero no es menor la preocupación por el rápido crecimiento demográfico de la zona, sobre todo en las catorce ciudades hermanas (se calculan casi 20 millones de habitantes en la región para el año 2020). El mayor problema de estas ciudades ha sido su crecimiento (hablamos de 2003) descontrolado y sin una dirección común, lo que ha provocado enormes demandas de suelo y de energía, con toda una serie de problemas derivados del creciente congestionamiento de tráfico, los ingentes depósitos de basura incontrolados y, casi siempre, sin tratamiento, además de un alarmante número de emergencias por vertidos químicos. A ello se unirían los problemas que acucian a la población rural, expuesta de forma permanente a polvo en suspensión y de pesticidas, así como los problemas derivados de la falta de tratamiento de las aguas. Todo ello causa una alarmante desproporción respecto a otras regiones en problemas de salud, sobre todo enfermedades respiratorias y por consumo de aguas contaminadas.

En estas circunstancias, el programa marco se plantea seis objetivos fundamentales:

1.- Reducir la contaminación del aire

2.- Reducir la contaminación del agua

3.- Reducir la contaminación de los suelos

4.- Mejorar la salud ambiental

5.- Reducir la exposición a sustancias químicas y peligrosas

6.- Mejorar el desempeño ambiental (SEMARNAT).

El informe se basa en el estudio de 23 indicadores relacionados con los puntos anteriores y organizados en seis apartados (región fronteriza México-Estados Unidos, de carácter demográfico; agua; agua; aire; suelo; preparación y respuesta a emergencias y, finalmente, aplicación y cumplimiento de la ley). Es interesante observar que en el propio informe queda explícitamente manifestado el problema de no tener unas fuentes comunes para la recogida de datos).

Como no se trata de hacer un análisis exhaustivo del informe de 2005, baste decir que, si bien se detectan problemas en todos los sectores analizados, hay un cierto optimismo en cuanto a resultados, aunque estos no son comparables con estudios hechos por instituciones ajenas a ambos gobiernos y, en consecuencia, a juzgar por las denuncias que cada día aparecen en la prensa, habría que reducir bastante dicho optimismo.

En la conclusión del informe se anuncia otro para 2008 en el que se recoja la actividad realizada en la primera mitad del programa (2003-2007). Dicho informe se ha realizado también y se ha publicado en red, como se prometía en el de 2005. Básicamente es una exposición de logros, pero, en cualquier caso, ambos informes dan una idea bastante clara de la situación fronteriza en el apartado medioambiental. Cabe señalar que ya hay un borrador para el programa Frontera 2020.

La visión que la Conferencia Legislativa Fronteriza tiene de la región no es tan optimista como la que se da en los dos informes Frontera 2012. En primer lugar, señala que los aspectos referidos a la salud son muy preocupantes en la región, con aumento de enfermedades como consecuencia de la contaminación por humos de camiones y coches particulares y sus largas esperas en la frontera, aunque se hayan construido «carriles rápidos para camiones de carga acelerada». Los casos de asma infantil crecen en números alarmantes, especialmente en el área Tijuana-San Diego. Por otro lado, la falta de agua potable, frente al muy optimista informe de la CONAGUA (Comisión Nacional del Agua, de México), sobre todo en áreas rurales, hace que las enfermedades derivadas, como fiebres tifoideas y disentería tengan también cifras alarmantes. Se constata desde la CLF que crece el número de visitas a emergencias y disminuye la actividad preventiva, sobre todo entre jóvenes y ancianos. 
Tampoco los investigadores están contentos con algunos de los resultados. Ensenada. net se hace eco de la noticia siguiente, publicada el 6 de octubre de 2011:

«La emanación de gases de efecto invernadero en los estados fronterizos se ha convertido en una de las principales preocupaciones de las autoridades ambientales de Estados Unidos y México. De acuerdo a María Elena Giner, Administradora y Gerente de la Comisión de Cooperación Ecológica Fronteriza (COCEF), un estudio reciente indica que actualmente los seis estados fronterizos mexicanos generan el $22 \%$ de los gases de efecto invernadero a nivel nacional. Para el 2025 esta generación será del 32\% del total nacional pero con el $21 \%$ de la población por lo cual se trabaja en distintos temas binacionales para reducir estas emisiones.»

Sin embargo, a través de la CLF se puede ver que los intentos porque la región fronteriza funcione como tal son importantes. Dado el tamaño de la región fronteriza, uno de los aspectos destacados es el funcionamiento de subregiones dentro del conjunto. Así lo demuestran los tres foros regionales fronterizos de desarrollo económico impulsados por el Comité de Desarrollo Económico. El primero de ellos, en septiembre de 2005, entre los estados de Texas, Nuevo México, Chihuahua, Coahuila, Nuevo León y Tamaulipas. El segundo, en febrero de 2006, entre California y Baja California y, el tercero, en agosto del mismo año, entre Arizona y Sonora. Eso sí, no se recogen nuevos encuentros con estas características.

En los tres, los asuntos tratados son siempre de cooperación transfronteriza, con temas (sin agotarlos) como cruces fronterizos inteligentes para el siglo XXI, corredores de alta prioridad en México-Estados Unidos, estrategias regionales para promover comercio de valor agregado, planeación binacional y financiamiento de infraestructuras, manufactura transfronteriza en el siglo XXI o asegurando y facilitando el comercio fronterizo, entre otros.

Mayor interés tiene, para ver el trabajo de la Conferencia Legislativa Fronteriza, el trabajo que dos veces al año (la última, número 24, celebrada en Saltillo, Coahuila, en octubre de 2011) se realiza en las «Reuniones Legislativas» de la misma. A lo que hay que sumar las declaraciones que acompañan a cada una de las reuniones y en las que se destacan los aspectos más significativos de las mismas. Los temas tocan todos los asuntos en los que la CLF tiene competencias, por lo que van desde cuestiones ambientales a otros relacionados con la inmigración ilegal o el comercio, por señalar algunos.

En la XIII Reunión de la CLF (mayo de 2006 en Monterrey) se hace, por ejemplo, la siguiente declaración, animando a los gobiernos federales de México y Estados Unidos a buscar soluciones a la inmigración ilegal:

«1.- El establecimiento de mecanismos binacionales que promueven la migración legal, ordenada, segura, y humana.

2.- Políticas publicas y reformas que establezcan las condiciones necesarias en México y los Estados Unidos que mitiguen la inmigración ilegal y promuevan integración económica para América del Norte.

3.- Colaboración estratégica entre dependencias de seguridad publica en todos los niveles de gobierno México - Estados Unidos para proteger a emigrantes de organizaciones criminales y de contrabandistas.»

En definitiva, se trata de la gestión regional de la frontera por parte de los legisladores que componen la CLF y que facilite la mayor integración posible a ambos lados de la fron- 
tera, buscando las fórmulas que acerquen a dos comunidades separadas por la nacionalidad $\mathrm{y}$ con fuertes desequilibrios entre ambas.

\section{Los desacuerdos}

Es evidente, como ya se señalaba al inicio del artículo y es obvio para quienes siguen los acontecimientos fronterizos, que esta región está lejos de ser homogénea y que los problemas son mucho mayores que los puntos de unión, por más que se vean los esfuerzos por ambas partes, sobre todo cuando se trata de las relaciones entre estados, lejos de las políticas seguidas por ambos gobiernos federales.

Dejando al margen el asunto del tráfico de drogas y de armas, sobre el que habrá que hablar algo al tocar temas de seguridad y de comercio, el asunto de la emigración a Estados Unidos por ciudadanos mexicanos y por los llegados de América Central, que se convierten también en un problema para México, es un asunto fundamental entre los desacuerdos que afectan a esta región. Aquí son todas las partes implicadas, desde las críticas que el gobierno mexicano hace al estadounidense por el trato a sus ciudadanos, ingresados ilegalmente en el vecino del norte, hasta las que se hacen, incluidos muchos legisladores de ambos lados de la frontera, a las nuevas leyes contra la inmigración ilegal que, en los últimos tiempos, se han ido aprobando en algunos estados fronterizos o, incluso, lejos de la frontera.

Desde México se ve como un derecho el paso a los Estados Unidos y la posibilidad de convertirse en ciudadano de pleno derecho en ese país. Por supuesto, desde el gobierno de los Estados Unidos y de no pocos estados, esta pretensión no es posible, mucho más cuando ya se cuenta con más de diez millones de inmigrantes ilegales. El problema se ha hecho más grave con la crisis económica y la fácil y rápida denuncia de que los inmigrantes quitan los empleos, cada vez más escasos, a los nacionales. Como ya se ha visto con un ejemplo, son muchas las peticiones que hace la CLF a las autoridades federales de los Estados Unidos para que resuelva de manera adecuada el problema de la inmigración que tiene no pocas implicaciones económicas y sociales, sobre todo desde el punto de vista del Sur.

La EPA, en su versión del programa Frontera 2012, señala que es muy grave el problema del crecimiento acelerado y sin control de las metrópolis fronterizas. Como bien ha estudiado Isabel Rodríguez Chumillas, da lugar, entre otras cosas, a bolsas de infravivienda en todas las grandes capitales del sur, con toda suerte de problemas ambientales y de salud. Buena parte de esa población llega a la frontera en busca del paso al «otro lado», pero se tiene que quedar presa en esos espacios y aspirar, como mucho, a un trabajo mal remunerado en las maquiladoras, por otra parte, en reducción por los problemas de seguridad que muchas ciudades fronterizas tienen por la guerra al narcotráfico.

CNNExpansión, en noticia del 5 de diciembre de 2011, afirma:

«El sector maquilador en México, tradicionalmente fundamental para la economía, ha encendido las luces de alerta por la precaria situación que vive desde hace más de una década en la que no ha generado empleos, advierten investigadores.

Desde 2000, «la industria maquiladora que se ubica en la franja fronteriza del Norte del país no ha sido generadora de empleos. De hecho, en los últimos 11 años se han perdido más de 200,000 fuentes de trabajo,» dice la investigadora del Departamento de Estudios Sociales del Colegio de la Frontera Norte (Colef) con sede en Matamoros, Cirila Quintero.» 
Por otro lado, señala también Rodríguez Chumillas, se crean más y más urbanizaciones cerradas, por no decir acorazadas, con normativas internas propias y que provocan grandes desajustes en la organización territorial de esas áreas metropolitanas, a la vez que, se puede añadir, suman otros efectos no deseados que denuncia la EPA en su informe, la ocupación de grandes cantidades de suelo que consumen enormes volúmenes de energía y basuras, en general, mal tratadas y creadoras de nuevos focos de contaminación.

Dicha guerra provoca víctimas colaterales en muchos sentidos y por algunas de ellas se preocupan también en la CLF (dejando de lado las reclamaciones al gobierno mexicano por violaciones de los derechos de muchas personas inocentes o, incluso, culpables). La víctima de la que ahora hablo es el comercio, que tanto preocupa a los legisladores y empresarios, no sólo de la frontera. Para que el comercio transfronterizo siga creciendo a los ritmos que lo ha hecho desde la firma del Tratado de Libre Comercio de América del Norte, es necesaria la seguridad, desde luego, pero los legisladores de la región ven en ella también una causa de preocupación si las medidas se adoptan unilateralmente y que, por combatir el tráfico ilegal de personas y de mercancías, se obstaculice de forma muy perjudicial el intercambio de productos legales. En la página de la CLF se puede leer lo siguiente cuando se habla de cruces fronterizos:

«Este bloqueo potencial de la frontera representa una amenaza para los cientos de miles de millones de dólares en el comercio legal de cada año. El costo, el transito lento y ineficiente forzará a los consumidores y los proveedores a buscar en otros mercados más competitivos como China. Para agilizar el tránsito de comercio seguro y legítimo con los vehículos comerciales, la frontera debe ser la última línea de defensa, no la primera.» (Conferencia Legislativa Fronteriza).

Para evitar esto, la única vía de solución pasa por una marcada cooperación entre los dos gobiernos federales.

No es, sin embargo, el problema del control fronterizo el único que provoca desacuerdos y tensiones entre los dos países y que se reflejan en la región. Desde 1996, México y Estados Unidos mantienen una agria disputa sobre el paso a este último país de transportistas mexicanos. La prensa, una vez más, se hace eco de un problema que, para muchos, no tiene una solución próxima. Alto Nivel, con fecha 23 de mayo de 2011, publicó la siguiente noticia:

«Luego de 15 años de disputa, Estados Unidos y México firmarán un acuerdo en materia de transporte fronterizo, no obstante aquello, la Cámara Nacional del Autotransporte de Carga (Canacar) considera que la apertura real tardará mucho tiempo en que sea efectiva, consideró Refugio Muñoz López, director general del organismo. Y es que la firma definitiva se ve cada vez más cerca, luego de que el Departamento de Transporte de Estados Unidos (DOT, por sus siglas en inglés) ya entregara al gobierno de México las reglas para que los transportistas circulen en su territorio. Ahora, la autoridad mexicana estaría analizando la contraparte que deberán acatar ellos en nuestro país. «Sin embargo, eso sólo en el papel», dijo Muñoz López. Al respecto, recordó que algunos de los aspectos que obstaculizan el transporte de carga entre ambas naciones, es que en México se carece de la producción de combustible con bajo contenido de azufre, lo que a su vez inhibe la llegada de nuevas tecnologías.

Problema histórico. La disputa transportista se arrastra desde 1996 cuando el vecino país se niega a la posibilidad de abrir paso a los camiones mexicanos a su territorio. Ello originó represalias comerciales por parte de México ante 
el incumpliendo. En marzo de 2009, el gobierno empezó a aplicar aranceles a 89 productos manufactureros y agrícolas de EU, luego de que Barack Obama, cancelara el apoyo económico al programa Demostrativo de Transporte que permitía a camiones mexicanos y americanos transitar más allá de 40 kilómetros de las fronteras. Luego de diversas réplicas de ese estilo, en marzo pasado ambos países anunciaron un acuerdo que debería concretarse con la firma en septiembre próximo.»

Además del problema generado para los transportistas mexicanos, para la región fronteriza el asunto agrava los ya mencionados problemas de tráfico y agilidad en el paso de un país al otro, porque todas las mercancías que llegan cargadas por camiones mexicanos tienen que cambiar de cabeza tractora para pasar la frontera. Más lentitud, más problemas de tráfico y más contaminación, no mencionada específicamente en ninguno de los informes.

\section{A modo de conclusión}

La región fronteriza México-Estados Unidos es un espacio complejo y apasionante que ofrece grandes posibilidades para su estudio geográfico. Si bien se han señalado los problemas que muchos investigadores perciben en la actualidad porque el trabajo en esos lugares, especialmente del lado mexicano, es arriesgado por la inseguridad en que se vive, a lo que hay que sumar la falta de estadísticas y estudios que sirvan como base para los mismos, el hecho es que, desde los organismos oficiales, tanto federales como de los órganos de control de dicha región, se va ofreciendo una importante cantidad de información (por muy sesgada que se quiera ver, sobre todo por venir de ambos gobiernos) que permitiría profundizar más en las relaciones interregionales, así como en los muchos problemas que aún tiene planteados. El hecho de que se hable de una región internacional creada entre dos países con tan fuertes diferencias sociales y económicas hace que el estudio sea más interesante, aunque ya en la Unión Europea el funcionamiento de regiones transnacionales va abriendo nuevas puertas a la investigación. Sin embargo, en este último caso, las homogeneidades son mayores y existe una legislación común en aspectos muy significativos.

Las fronteras en casi todo el mundo siguen siendo un lugar de paso vigilado y, muchas veces, controvertido o en disputa. Entre México y Estados Unidos, es evidente, la frontera tiene mucho de cerrojo que impide o dificulta la circulación de personas, sobre todo de sur a norte, pero también es la línea a cuyos lados se ha creado una región, muy bien delimitada por el Acuerdo de La Paz, y que comparte una gran cantidad de intereses. Hasta el punto de no pocos autores consideran que el norte de México mira más a Estados Unidos, se relaciona mejor, sobre todo desde el punto de vista comercial, con ese país que con el resto de la República Mexicana.

Las drogas, el tráfico de personas y de armas, dificultan las relaciones y la actividad regional, así como numerosos asuntos pendientes aún no resueltos, incluso después de casi veinte años de tratado de libre comercio. O por causa del mismo. Por otra parte, numerosas instituciones públicas y privadas, a ambos lados de la frontera, luchan por consolidar unas relaciones que, al menos, han encontrado dos bases sólidas de unión, la protección ambiental y las actividades comerciales, sin olvidar que el sector norte de la región tiende a mexicanizarse (al menos a ser mucho más intercultural) por la cada vez más fuerte presencia de ciudadanos de este país en los estados fronterizos de Estados Unidos. El informe SEMARNAT/EPA señala el avance del español como lengua que, poco a poco, se acerca a cifras importantes. En Texas, por ejemplo, el 36\% de la población habla sólo español, pero hay otro $42 \%$ bilingüe. En la frontera con cifras más bajas, la californiana, entre unos 
y otros ya son la cuarta parte de la población. En definitiva, los cambios en la región son muy rápidos y conviene no perder de vista el proceso.

\section{Bibliografía}

ACUERDO DE LA PAZ (1983): http://www.epa.gov/usmexicoborder/docs/LaPazAgreement.pdf

BASSOLS BATALLA, A. (1998): Franjas fronterizas México-Estados Unidos. Tomo I, Dominio, conflicto y desintegración territoriales. Instituto de Investigaciones Económicas, UNAM. México, $297 \mathrm{pp}$.

CONFERENCIA LEGISLATIVA FRONTERIZA. http://www.borderlegislators.org/description_esp. htm

EPA (s.a.): U.S.-Mexico Border 2012. http://www.epa.gov/usmexicoborder/index.html

EPA (2008): Programa ambiental México-Estados Unidos: Frontera 2012. Perfeccionamiento a mitad de periodo (2008-2012).http://www.epa.gov/Border2012/docs/B2012_New_Objectives_SPN.pdf

INDUSTRIA ECONOMICS, INCORPORATED y ROSS \& ASSOCIATES ENVIRONMENTAL CONSULTING, LTD. (2007): Análisis de emisiones de diesel en la región fronteriza de México y Estados Unidos. IEC. Cambridge, Mass., 108 pp.

MENDOZA, C. (2001): «Sociodemografía de la región fronteriza México-Estados Unidos: tendencias recientes», en Papeles de Población, $\mathrm{n}^{\circ}$ 30, Universidad Autónoma del Estado de México, pp. 31-63.

PAPADODIMA, Z. (2011): «Las fronteras regionales: la materia de migración en la geopolítica contemporánea», en Cuadernos Geográficos, Universidad de Granada, n 48, pp. 189-205.

QUINTERO NÚÑEZ, M., SÁNCHEZ LÓPEZ, E. y COLLINS, K. (2005): Desarrollo y medio ambiente de la región fronteriza México-Estados Unidos: Valles de Imperial y Mexicali. Porrúa. México, 509 pp.

RODRÍGUEZ CHUMILLAS, I. (2007): «Paisajes de frontera», en MÉNDEZ, E. Y RODRÍGUEZ CHUMILLAS, I.: Paisajes y arquitecturas de la exclusión. Universidad Autónoma de Madrid. Madrid pp. 97-127.

SEMARNAT (2005): Programa ambiental México-Estados Unidos: Frontera 2012. Situación ambiental en la región fronteriza. $\mathrm{http}$ :/www.semarnat.gob.mx/informacionambiental/publicaciones/ Publicaciones/frontera2012_espanol.pdf

VÁSQUEZ GALÁN, T. y CUEVA LUNA, E. (2001): «Saludo y maternidad en la frontera MéxicoEstados Unidos. El caso de la región Matamoros-Brownsville», en Revista Frontera Norte, $\mathrm{n}^{\circ}$ especial, El Colegio de la Frontera Norte, México, pp. 243-281.

VÁZQUEZ RUIZ, M. A. (1999): «Región e integración fronteriza México-Estados Unidos», en Región y Sociedad, El Colegio de Sonora, n 17, pp. 113-143. 